\title{
Experiments on spontaneous vortex formation in Josephson tunnel junctions
}

Monaco, R.; Aarøe, Morten; Mygind, Jesper; Rivers, R.; Koshelets, V.

Published in:

Physical Review B Condensed Matter

Link to article, DOI:

10.1103/PhysRevB.74.144513

Publication date:

2006

Document Version

Publisher's PDF, also known as Version of record

Link back to DTU Orbit

Citation $(A P A)$ :

Monaco, R., Aarøe, M., Mygind, J., Rivers, R., \& Koshelets, V. (2006). Experiments on spontaneous vortex formation in Josephson tunnel junctions. Physical Review B Condensed Matter, 74(14), 144513.

https://doi.org/10.1103/PhysRevB.74.144513

\section{General rights}

Copyright and moral rights for the publications made accessible in the public portal are retained by the authors and/or other copyright owners and it is a condition of accessing publications that users recognise and abide by the legal requirements associated with these rights.

- Users may download and print one copy of any publication from the public portal for the purpose of private study or research.

- You may not further distribute the material or use it for any profit-making activity or commercial gain

- You may freely distribute the URL identifying the publication in the public portal

If you believe that this document breaches copyright please contact us providing details, and we will remove access to the work immediately and investigate your claim. 


\title{
Experiments on spontaneous vortex formation in Josephson tunnel junctions
}

\author{
R. Monaco* \\ Istituto di Cibernetica del CNR, 80078, Pozzuoli, Italy \\ and Unita INFM-Dipartimento di Fisica, Università di Salerno, 84081 Baronissi, Italy \\ M. Aaroe ${ }^{\dagger}$ and J. Mygind \\ Department of Physics, B309, Technical University of Denmark, DK-2800 Lyngby, Denmark \\ R. J. Rivers $\$$ \\ Blackett Laboratory, Imperial College London, London SW7 2AZ, United Kingdom
}

V. P. Koshelets ${ }^{\S}$

Institute of Radio Engineering and Electronics, Russian Academy of Science, Mokhovaya 11, Building 7, 125009, Moscow, Russia

(Received 22 June 2006; revised manuscript received 6 August 2006; published 18 October 2006)

\begin{abstract}
It has been argued by Zurek and Kibble that the likelihood of producing defects in a continuous phase transition depends in a characteristic way on the quench rate. In this paper we discuss an improved experiment for measuring the scaling exponent $\sigma$ for the production of single fluxons in annular symmetric Josephson tunnel junctions. We find $\sigma \simeq 0.5$ and show how this can arise from the Kibble-Zurek scenario. Further, we report accurate measurements of the temperature dependence of the junction gap voltage, which allow for precise monitoring of the fast temperature variations during the quench.
\end{abstract}

DOI: 10.1103/PhysRevB.74.144513 PACS number(s): 74.50.+r, 74.81.Fa, 11.10.Wx, 67.40.Vs

\section{INTRODUCTION}

The experiments that we describe here are part of a program ${ }^{1-3}$ designed to see whether continuous phase transitions in superconductors proceed as fast as they can, in the sense that the resulting domain structure reflects causal horizons. Although, under adiabatic change, correlation lengths do diverge at the critical temperature $T_{c}$, in reality causality prevents any lengths diverging since transitions take place in a finite time. This seemingly simple observation is not of purely academic interest, since causality is universal, and the same is equally true for the early universe with its anticipated rich sequence of symmetry breaking. Constraints imposed by causal horizons in the early universe are known to have observable consequences ${ }^{4}$ and, in addition to being of interest in its own right, one motive for our, and related, work is to see how transitions in condensed matter systems can mimic this behavior.

The suggestion that causality constrains the domain structure of the early universe after a transition was made by Kibble $^{4}$ in 1976, although the original conjecture that the scale was set by fluctuations at the Ginzburg temperature was subsequently seen to be incorrect. A later conjecture by Kibble $^{5}$ with a somewhat different approach was paralleled by Zurek ${ }^{6,7}$ for condensed matter systems, in what we term the Kibble-Zurek (KZ) scenario, and it is this, based upon the assumption that systems change as fast as they can, that concerns us here. In particular, if transitions are frustrated, topological defects (vortices, monopoles, etc.) arise to mediate the different ground states of the theory. Their density will be related to the nature of the domain structure present and be constrained by causality in turn. The relevance of this is that defects are, in principle, readily countable, permitting us to check this proposition. Without taking the parallels any further, we note that all reasonable models for the early universe show frustration on cooling. ${ }^{8}$
We would expect that the faster the quench through the transition the more defects we would see. Whatever the details, and there are several ways to estimate the defect density in the KZ picture, all predict characteristic scaling behavior in the quench time $\tau_{Q}$ (the inverse quench rate), defined in terms of the temperature $T$ by

$$
\frac{T_{C}}{\tau_{Q}}=-\left.\frac{d T}{d t}\right|_{T=T_{C}} .
$$

Specifically, if $\bar{\xi}$ is the separation of defects at the time of their production then it is predicted to scale with $\tau_{Q}$ as

$$
\bar{\xi} \approx \xi_{0}\left(\frac{\tau_{Q}}{\tau_{0}}\right)^{\sigma}
$$

where $\xi_{0}$ is, most simply, the cold correlation length, and $\tau_{0}$ the relaxation time of the long-wavelength modes.

The scaling exponent $\sigma>0$ is, in the mean-field approximation, determined from the static mean-field critical exponents and whether the dynamics is largely wavelike (early universe) or dissipative (condensed matter). That is, universality classes of continuous adiabatic transitions lead to identical scaling behavior of domains as the cooling rate is changed.

For Josephson tunnel junctions (JTJs) the topological defect is a fluxon, i.e., a supercurrent vortex carrying a single quantum of magnetic flux $\Phi_{0}=h /(2 e)$ in the plane of the oxide layer between the two superconductors that make up the JTJ. Our experiments, summarized in part in Ref. 3, that we detail below, show that the spontaneous production of fluxons at a temperature quench does, indeed, scale with the quench rate.

This is a considerable achievement since, in general, it has proven surprisingly difficult to establish the scaling be- 
havior of Eq. (2) experimentally. In fact, although there have been many experiments that have been performed to test the $\mathrm{KZ}$ picture that are commensurate with it or, when not, explicable in its framework, ${ }^{9-18}$ our experiments are sensitive enough to show unambiguous scaling behavior.

It is worth commenting briefly on how using Josephson junctions enables us to avoid the main problems that have befallen other experiments with superfluids and superconductors which, superficially, look simpler and more direct than ours. The first problem, which besets superfluids, is that of relating the density of defects at the time of measurement to the density at the time of formation, as (2) requires. This was a particular problem for superfluid ${ }^{4} \mathrm{He}$, leading to the null experiments of Ref. 12 after the spurious results of Ref. 11. Although this is avoided in spontaneous vortex production in superfluid ${ }^{3} \mathrm{He}$, because of the ability to count vortices more accurately in this case, the fact that ${ }^{3} \mathrm{He}$ is heated by its disintegration under bombardment by soft neutrons ${ }^{13,14}$ means that the fixed rate of the nuclear reaction constrains the effective cooling rate. Even though, in principle, the pressure dependence of the coherence length and heat capacity permit a demonstration of scaling, it has not been possible to extract scaling exponents explicitly from the experiments as they stand.

Both of these problems appear to be avoided with planar superconductors, where flux is conserved, and where a wide range of cooling rates can be implemented. ${ }^{15,16}$ However, since only net flux can be measured, effectively halving the scaling exponent, its expected value is so small that it takes an even wider range of quench rates than are readily available in order to show scaling robustly. This is compounded by the fact that not only are the systems noisy, but Hindmarsh and Rajantie ${ }^{19}$ have shown that the freezing in of pure magnetic flux gives an additional contribution to the flux density anticipated from Eq. (2), which complicates the issue. While JTJs possess the virtues of superconductors, they avoid this latter problem since there is no counterpart of the Hindmarsh-Rajantie mechanism for flux produced in a narrow slit.

However, we do have a potential problem in that our samples are so small that we expect to see no more than one (conserved) fluxon in each annulus in each measurement. Although noise is minimized, Eq. (2), couched in the language of causal horizons, is not designed for systems, such as ours, that are much smaller than them. Instead, we propose that the probability $f_{1}$ for spontaneously producing one fluxon in the thermal quench of a symmetric annular JTJ of circumference $C<\bar{\xi}$ should scale with the quench time $\tau_{Q}$ (the inverse quench rate) as

$$
f_{1} \simeq \frac{C}{\bar{\xi}}=\frac{C}{\xi_{0}}\left(\frac{\tau_{Q}}{\tau_{0}}\right)^{-\sigma}
$$

where the scaling exponent $\sigma$ depends on the nature of the junction. We shall motivate Eq. (3) in our concluding sections but, for the moment, we take it as having the same origins as Eq. (2) and include it under the KZ umbrella.

We conclude this section by putting our present experiments in the context of our previous ones. In Refs. 1 and 2 we reported our proof-of-principle experiment with JTJs, to test the scaling behavior of (3). The experiment consisted of taking an annular JTJ isolated from its liquid hellium (LHe) surroundings and making it undergo a forced phase transition by heating it above its superconducting critical temperature and letting it cool passively back toward the LHe temperature with no external current or magnetic field. In common with all our experiments, any trapped fluxon can be detected by the appearance of a current peak in the $I-V$ characteristic of the JTJ, as will be explained later.

To derive $\sigma$ two of us had argued earlier ${ }^{20}$ that the relevant causality is provided by the finite velocity of electromagnetic waves in the JTJ, the Swihart velocity. ${ }^{21,22}$. Under the idealistic assumptions of (a) weak coupling of the superconductors and (b) exact critical slowing of the Swihart velocity at the critical temperature $T=T_{c}$, we predicted $\sigma$ $=0.25 .{ }^{20}$ The experiment was successful, with $\sigma$ commensurate with scaling behavior (3) with this value of $\sigma$.

The experiments performed subsequently, that we shall describe in this paper, have forced us to revise our assumptions. In our recent Letter ${ }^{3}$ we showed different scaling behavior for the spontaneous production of fluxons, in which $f_{1}$ was indeed seen to clearly follow an allometric dependence on $\tau_{Q}$, but with a scaling exponent $\sigma=0.5$. We discuss this experiment in more detail in the subsequent sections and confirm this behavior with data from new samples (see Fig. 4). The discrepancy between the observations of the early and later experimental values of $\sigma$ may be less than it looks at first sight, given the high accuracy of the latter and the relative scatter of the former. However, from a theoretical viewpoint, it may also lie in the fact that we need to take into account the consequences of finite size and the nature of the fabrication of the junction, which differs between the two sets of samples. In this regard realistic condensed matter systems cannot match the early universe for their extension and uniformity, upon which (2), with $\sigma$ based simply on the usual critical exponents, is predicated. Again we postpone a proper discussion of these issues, and the calculation of $\sigma$, to the concluding sections of this paper.

However, before then we shall describe the nature of the samples and the experimental setup, the use of the JTJ as its own thermometer to measure quench times, and then the results confirming the scaling behavior of (3).

\section{THE SAMPLES}

To begin with some generalities, the annular JTJs (AJTJs) that we have used are high-quality $\mathrm{Nb} / \mathrm{Al}-\mathrm{AlO}_{x} / \mathrm{Nb}$ JTJs fabricated on silicon substrates using the trilayer technique in which the junction is realized in the window opened in a $\mathrm{SiO}$ insulator layer. The so-called idle region, i.e., the overlapping of the wiring layer onto the base electrode, was about $3 \mu \mathrm{m}$ for all the junctions. The thicknesses of the base, top, and wiring layer were 200,80 , and $400 \mathrm{~nm}$, respectively. Details of the fabrication process can be found in Ref. 23. The samples were fabricated at the Superconducting Electronics Laboratory of the Institute of Radio Engineering and Electronics of the Russian Academy of Science in Moscow, while the measurements were carried out at the Physics De- 


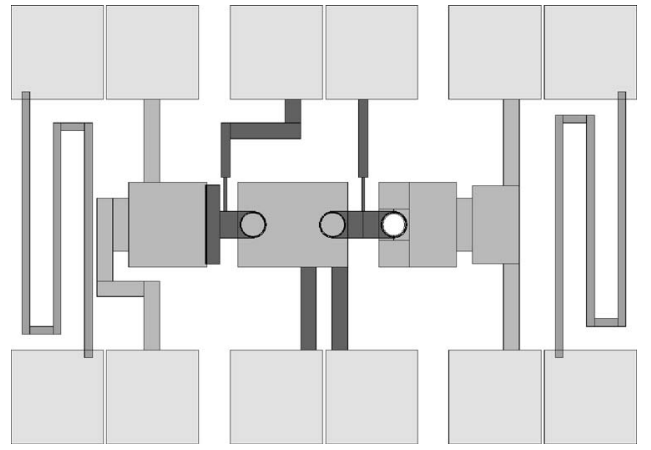

FIG. 1. Layout of the $4.2 \times 3 \mathrm{~mm}^{2} \mathrm{Si}$ chip containing four series-biased $\mathrm{Nb}-\mathrm{Al} / \mathrm{AlO}_{x} / \mathrm{Nb}$ Josephson tunnel junctions. It integrates three ring-shaped junctions having a mean circumference $C$ $=500 \mu \mathrm{m}$ and a width $\Delta r=4 \mu \mathrm{m}$, one $4 \times 500 \mu \mathrm{m}^{2}$ overlap-type linear junction, and two meander line resistive Mo strips used for heating.

partment of the Danish Technical University in Lyngby (DK). All the experiments performed to date have been carried out on AJTJs with a mean circumference $C=500 \mu \mathrm{m}$ and a width $\Delta r=4 \mu \mathrm{m}$. AJTJs with larger circumferences have been fabricated, but we have yet to use them.

The chip layout developed for the $\mathrm{KZ}$ experiment is shown in Fig. 1. It integrates three ring-shaped junctions having a mean circumference $C=500 \mu \mathrm{m}$ and a width $\Delta r$ $=4 \mu \mathrm{m}$ and one $4 \times 500 \mu \mathrm{m}^{2}$ overlap-type linear junction. The four JTJs are biased in series. The rightmost AJTJ was obtained by the superposition of two superconducting rings, as depicted in Fig. 2(a), while the two AJTJs in the layout middle were realized by the superposition of a ring-shaped top electrode over a superconducting plane, as shown in Fig. 2(b). Here we anticipate that this difference in the sample topology did not affect the measured spontaneous defect production.

For this experiment a faster and more reliable heating system was required. This was achieved by integrating a meander line $50 \mu \mathrm{m}$ wide, $200 \mathrm{~nm}$ thick, and $8.3 \mathrm{~mm}$ long of Mo resistive film in either end of the $4.2 \times 3 \times 0.35 \mathrm{~mm}^{3} \mathrm{Si}$ chip containing the $\mathrm{Nb} / \mathrm{AlO}_{x} / \mathrm{Nb}$ trilayer JTJs (also shown in Fig. 1). These resistive elements have a nominal dc resistance of $50 \Omega$ at LHe temperatures and, due to their good adhesion with the substrate, are very effective in dissipating heat in the chip. In fact, voltage pulses a few microseconds long and a few volts high applied across the integrated heater provided quench times as low as $1 \mathrm{~ms}$, which is more than two orders of magnitude smaller than for the previous situation. ${ }^{1,2}$ The on-chip heaters are able to sustain many thousands of pulses as well as $1 \mathrm{~V}$ continuous bias without any appreciable change of their electrical resistance.

\section{THE EXPERIMENTAL SETUP}

A detailed description of the experimental setup has been given in Ref. 2. Briefly, the chip with the AJTJs is mounted on a $\mathrm{Cu}$ block enclosed in a vacuum-tight can immersed in the liquid $\mathrm{He}$ bath. A Ge thermometer anchored to the $\mathrm{Cu}$ block allowed for precise measurements of the chip static or
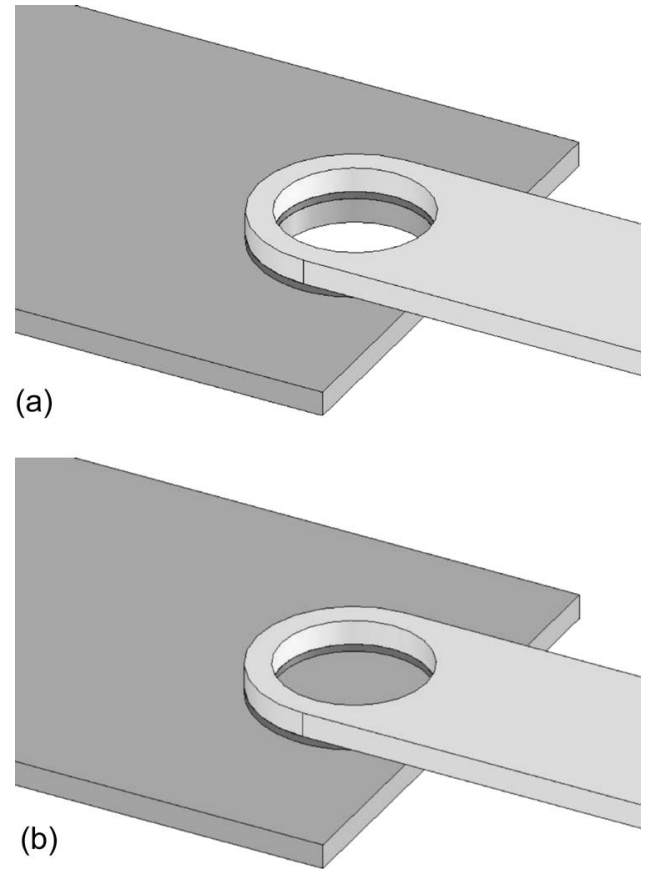

FIG. 2. Sketch (not in scale) of two geometries for the annular Josephson tunnel junctions used for the Kibble-Zurek experiment. The junction base and top electrodes are shown in dark and light gray, respectively. In (a) the annular junction is obtained by the superposition of two superconducting rings, while in (b) it is realized by the superposition of a ring-shaped superconducting top electrode over a superconducting plane base electrode.

slowly changing temperature. The junction itself is used for quickly changing temperature measurements, as will be explained in the next section, but with lower accuracy. The chip was heated above the AJTJ critical temperature by a voltage pulse applied to one or both of the integrated meander line heaters. Then the heat is removed from the chip both through the thermal contact with the $\mathrm{Cu}$ block and by He exchange gas inside the can. For all the experiments performed so far the He exchange gas pressure was fixed at a value of about 7 mbar, which makes the heat flow through the copper base plate predominant.

In order to minimize thermal gradients during the thermal cycles, particular care was taken to have clean and flat contact surfaces of both the chip and the Cu block; further, the voltage pulses were applied simultaneously to both the onchip heaters. A tiny layer of low-temperature grease was spanned underneath the chip to improve the thermal contact.

At the end of each cycle the possible spontaneously generated fluxons are static. An external current supplied to the AJTJ sets the fluxons (if any) in motion around the annulus and quantized voltages develop across the junction itself. In other words, we count the number of produced fluxons by a careful inspection of the junction current-voltage (IV) characteristic. (Due to the annihilation of a fluxon-antifluxon pair, this idea works well as long as the chances to spontaneously generate two fluxons are small.) Fluxon motion at $4.2 \mathrm{~K}$ is very unstable in our samples due to very low junction losses; therefore the $I V$ characteristic was better inspected at higher temperatures where larger losses stabilize 
the fluxon motion. For our sample the optimal temperature to look for fluxons moving around the ring was in a range between 6 and $7 \mathrm{~K}$.

Quenching experiments were carried out in a double $\mu$-metal shielded cryostat and the sample holder can was surrounded by a superconducting $\mathrm{Pb}$ shield. In turn the chip holder inside the can was enclosed in a cryoperm shield and in one more superconducting $\mathrm{Pb}$ shield. The transitions from the normal to the superconducting states were performed with no current flowing in the heaters and the thermometers. During the quench the JTJ was also electrically isolated: in fact, both the junction voltage and current leads were open during the whole thermal cycle. Finally, all the measurements have been carried out in an electromagnetically shielded environment.

In order to run batches of several thousand equal thermal cycles with given parameters, automatization of thermal cycles was implemented by means of a switching unit controlled by a GPIB interface, which also allowed for much more robust statistics to be achieved. At the end of each thermal quench the junction $I V$ characteristic is automatically digitally acquired, converted, and stored. Then at the end of each batch with a given value of $\tau_{Q}$ an algorithm has been developed for the analysis of the large amount of $I V$ characteristics and the automatic detection and counting of the spontaneously trapped fluxons.

\section{DETERMINING THE QUENCHING TIME}

The quench time $\tau_{Q}$ was continuously varied over more that four orders of magnitude (from $20 \mathrm{~s}$ down to $1 \mathrm{~ms}$ ) by varying the width and the amplitude of the voltage pulse across the integrated resistive elements. In order to estimate the quenching time $\tau_{Q}$ we use the observation by Thouless ${ }^{24}$ that the junction itself acts as a thermometer, as long as its temperature is below the critical temperature. This permits high accuracy in measuring $\tau_{Q}$.

Specifically, the temperature dependence of the gap energy $\Delta$ in a strong-coupling superconductor, such as $\mathrm{Nb}$,

$$
\frac{\Delta(T)}{\Delta(0)}=\tanh \frac{\Delta(T)}{\Delta(0)} \frac{T_{c}}{T},
$$

also applies to the junction gap voltage $V_{g}$ which is proportional to $\Delta$. An experimental demonstration of Eq. (4) in $\mathrm{Nb} / \mathrm{Nb}$ tunnel junctions having native oxide was first evidenced in $1976,{ }^{25}$ well before the development of the trilayer technique used here, which, by exploiting the more compact $\mathrm{Al}$ oxide, allows for a higher quality and a more stable tunnel barrier.

Equation (4) can be manipulated: considering that $\operatorname{arctanh} x=\frac{1}{2} \ln \frac{1+x}{1-x}$ as long as $x^{2}<1$ and approximating $\ln (1 \pm x) \simeq \pm x-x^{2} / 2 \pm x^{3} / 6$, we easily get

$$
\frac{\Delta(T)}{\Delta(0)} \simeq\left[1-\left(\frac{T}{T_{c}}\right)^{4}\right]^{2 / 3} .
$$

In our samples we have found that, provided the JTJ is current biased at about $20-25 \%$ of the total current jump at the gap voltage, the junction temperature can be monitored to a

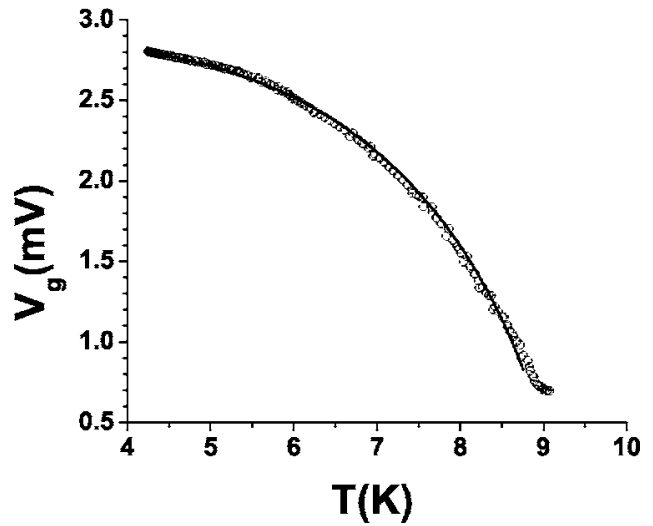

FIG. 3. The temperature dependence of gap voltage $V_{g}$. The open circles are the experimental data with the junction biased at $25 \%$ of the total current jump at the gap voltage; the solid line is the prediction that follows from Eq. (4). The fit yields $T_{C}$ $=9.12 \pm 0.04 \mathrm{~K}$ and $V_{g}(T=0)=2.89 \pm 0.02 \mathrm{mV}$.

high degree of accuracy and speed by resorting to Eq. (4). This is shown in Fig. 3 where the experimental values (open circles) of the junction gap voltage $V_{g}(T)=2 \Delta(T) / e$ are plotted at different values of the temperature as measured by a calibrated Ge thermometer. The solid line is the prediction that follows from Eq. (4) with fitting parameters $T_{C}$ $=9.12 \pm 0.04 \mathrm{~K}$ and $V_{g}(0)=2.89 \pm 0.02 \mathrm{mV}$, i.e. $2 \Delta(0) / k_{B} T_{c}$ $\simeq 3.73 \pm 0.03$. As a result, Eq. (4) can be used efficiently to estimate the junction temperature to a high degree of accuracy for $V_{g}(T)>1 \mathrm{mV}$, i.e., $T<8.5 \mathrm{~K}$. Above $8.5 \mathrm{~K}$ the experimental data for the gap voltage saturate to a finite temperature-independent value corresponding to the product of the JTJ normal resistance and the bias current. This way of monitoring the system temperature is particularly convenient when the temperature changes rapidly and a complete thermal cycle occurs on a millisecond time scale or even shorter. However, the overall temperature accuracy $\delta T=\left|\frac{d T}{d V_{g}}\right| \delta V_{g}$ cannot be smaller than $2 \mathrm{mK}$ due to a voltage accuracy $\delta V_{g}$ of about $2 \mu \mathrm{V}$ on a fast digital oscilloscope.

Assuming the chip exchanges heat mainly through a massive copper base plate (with thermal constant $\tau_{1}$ ) which in turn exchanges heat with the surrounding helium gas (with thermal constant $\tau_{2}$ ), the thermal relaxation during the thermal quench has been fitted by a double exponential decay of the form

$$
T(t)=T_{f i n}+\Delta T_{1} \exp \left(-\frac{t-t_{0}}{\tau_{1}}\right)+\Delta T_{2} \exp \left(-\frac{t-t_{0}}{\tau_{2}}\right)
$$

with $\Delta T_{1}, \Delta T_{2}, \tau_{1}$, and $\tau_{2}$ fitting parameters. $T_{\text {fin }}$ and $t_{0}$ are known from the experiments. If the time origin is triggered by the voltage pulse, then $t_{0}$ corresponds to the time at which the pulse ends. Once the parameters in Eq. (5) are determined or measured, the quenching time $\tau_{Q}$ can be inferred from its definition Eq. (1), after Eq. (5) has been extrapolated up to the critical temperature $T_{C}$. At the end of this process of fitting and extrapolation, $\tau_{Q}$ is known to an overall accuracy of about $\pm 10 \%$. 


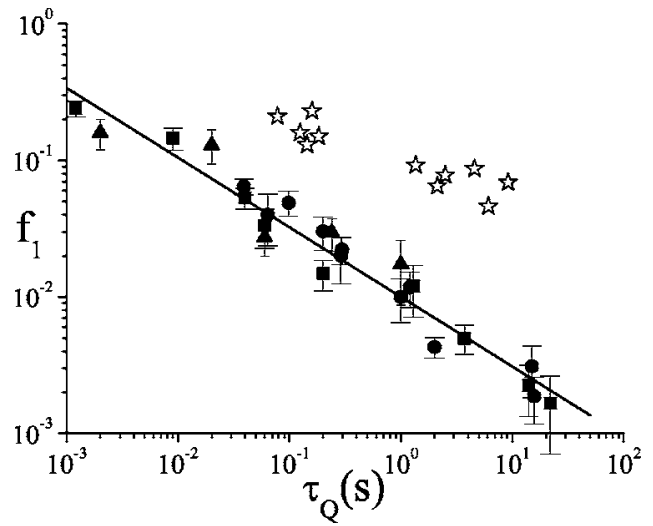

FIG. 4. Log-log plot (base 10) of the measured frequency $f_{1}$ of trapping single fluxons versus the quenching time $\tau_{Q}$. Each point corresponds to many thermal cycles, closed squares for sample 22-4, closed triangles for sample 08-3, and closed circles for sample 08-4. The vertical error bars give the statistical error while the relative error bars in $\tau_{Q}$ amounting to $\pm 10 \%$ are as large as the dots' width. The solid line is the fit of all data to an allometric relationship $f_{1}=a \tau_{Q}^{-b}$ which yields $a=0.01 \pm 10 \%$ (taking $\tau_{Q}$ in seconds) and $b=0.51 \pm 5 \%$. The open stars represent the data obtained in a previous experiment (Refs. 1 and 2).

\section{THE SCALING MEASUREMENTS}

The experimental results reported here refer to three identical AJTJs belonging to two different chips made within the same batch having a critical current density $J_{c}(0)$ $\simeq 60 \mathrm{~A} / \mathrm{cm}^{2}$ yielding a Josephson penetration depth $\lambda_{J}(0)$ $\simeq 50 \mu \mathrm{m}$. Assuming $\alpha^{\prime}=\alpha=3.5$ in Eq. (11), this leads to a value of $\xi_{0} \simeq 17 \mu \mathrm{m}$. For all samples the high quality has been inferred by a measurement of the $I V$ characteristic at $T=4.2 \mathrm{~K}$. Due to the very high reliability of the fabrication line the sample tunnel barriers have the same geometrical and electrical parameters. However, to distinguish them we will name them after their chip and junction numbers, that is, we have samples 08-3, 08-4, and 22-4. Samples 08-3 and 08-4 belonged to the same chip. Samples 08-4 and 22-4 had the geometry sketched in Fig. 2(a), while sample 08-03 had the geometry shown in Fig. 2(b). The solid symbols in Fig. 4 show on a log-log plot (base 10) the measured frequency $f_{1}=n_{1} / N$ of single-fluxon trapping, obtained by quenching the samples $N$ times for each value of a given quenching time $\tau_{Q}, n_{1}$ being the number of times that the inspection of the low-temperature AJTJ current-voltage characteristics at the very end of each thermal cycle showed that one defect was spontaneously produced. $N$ ranged between 100 and 5000 and $n_{1}$ was never smaller then 10 , except for some of the rightmost points $\left(\tau_{Q}>10 \mathrm{~s}\right)$ for which $n_{1} \geq 3$. All samples have undergone a total of more than 100000 thermal cycles without any measurable change of their electrical parameters. The vertical error bars give the statistical error $f_{1} / \sqrt{ } n_{1}$. The relative error bars in $\tau_{Q}$ amounting to $\pm 10 \%$ are as large as the dot's width.

It is quite evident that the dependence of the trapping frequency on the quenching time is the same for all three AJTJs independently of the geometry of their base electrodes. Careful measurements of the junction $I V$ characteris- tic during the normal-to-superconducting N-S transition indicate that the junction critical temperature might differ from the critical temperature of the base electrode film by no more than $10 \mathrm{mK}$, while the critical temperature of the wiring film, being twice thicker, exceeded the critical temperature of the base electrode by about $100 \mathrm{mK}$. In other words, at the time the Josephson effect is installed, the base electrode is only weakly superconducting and cannot exercise any shielding effect. This explains why we have not observed any difference between the data of junction 08-3 and those of junctions 08-4 and 22-4.

To test Eq. (3), we have fitted the data of all samples with the same allometric function $f_{1}=a \tau_{Q}^{-b}$, with $a$ and $b$ as free fitting parameters. A linear regression of $\log _{10} f_{1}$ vs $\log _{10} \tau_{Q}$, represented by the continuous line in Fig. 4, yields $a$ $=0.01 \pm 10 \%$ (taking $\tau_{Q}$ in seconds) and $b=0.51 \pm 5 \%$. Therefore the present experiment suggests that the scaling exponent is $\sigma=0.5$, rather than the value $\sigma=0.25$ suggested by our earlier attempt. ${ }^{1,2}$ For comparison, the data of this experiment are reported in Fig. 4 as open stars.

The shift in intercept (or, equivalently, prefactor $a$ ) between the two sets of data is to be expected. The AJTJs used in the first experiment, although of the same geometry as samples 08-4 and 22-4 [see Fig. 2(a)], had a Josephson current density $J_{c}$ about 60 times larger. As we shall reiterate later, this means a smaller Zurek length $\bar{\xi} \propto 1 / \sqrt{J}_{c}$, with a correspondingly greater likelihood of observing a fluxon. In other words, we have moved from a situation in which $C$ $<\bar{\xi}$ to $C \ll \bar{\xi}$. As we shall see, the value $a=0.01 \pm 10 \%$ (taking $\tau_{Q}$ in seconds) is 6-7 times larger than the predicted value. As a bound we only expect agreement in the overall normalization $a$ to somewhat better than an order of magnitude. Empirically, the different condensed matter experiments have shown that the ratio $a_{\text {observed }} / a_{\text {predicted }}$ varies widely from system to system; $O(1)$ for superfluid ${ }^{3} \mathrm{He},{ }^{13,14}$ very small for high- $T_{c}$ superconductors. ${ }^{16}$

Although the best fit to the stars alone is $\sigma=0.25$, when seen in conjunction with our new data, this value is not so compelling, due to the poor statistics and to the scattering of the stars. In retrospect, we do not exclude the possibility of systematic error in the earlier experiments. The trapping frequency $f_{1}$ increases when thermal cycling occurs in an externally applied magnetic field. Therefore an insufficient shielding of the earth's magnetic field might be the cause of the systematic shift upward of $f_{1}$ for increasing $\tau_{Q}$ in the first experiment. For the actual experiment we have taken a lot of precautions against the possibility of having a significant dc residual magnetic field. In fact, although its absolute value cannot be measured, we have checked that the measured trapping frequency did not change (i) by rotating the sample holder in the horizontal plane (which changes the sample orientation with respect to the direction of the earth's magnetic field) and (ii) by rotating the chip holder inside the sample holder, which is kept fixed (which changes the sample orientation with respect to the shields). Furthermore, detailed measurements have been carried out on the dependence of the trapping frequency on the strength of an external field applied perpendicular to the junction plane for different samples and for several values of the quenching time. 
Such data will be reported elsewhere, ${ }^{26}$ when we hope to discuss also the interplay between the Abrikosov vortices produced in the superconducting junction electrodes and the Josephson vortices produced in the junction barrier during the thermal quench. As far as the measurements presented in this work are concerned we made sure that for each value of the quenching time the corresponding trapping frequency lies at the minimum.

There is another plausible explanation for the different experimental findings to which we shall return in the next section. This is that the samples used in the two experiments, although constructed in the same fabrication line, might have slightly different proximity interfaces, which results in quite different temperature dependence of the critical current density near the transition temperature.

Whatever the reasons for the differences, the data of Fig. 4 resolve another issue. It could be argued that the causal behavior is determined by the phase ordering from the individual superconductors, with its own critical slowing down, rather than the Swihart velocity of the oxide. We could then adopt the results of Zurek ${ }^{6,7}$ for the spontaneous flux produced on quenching annuli of simple superconductors, but without having to worry about the flux trapping of Ref. 19. At the same level of approximation we would have predicted $\sigma=0.25$, which is manifestly not the case. It might have been thought that the experiment of Ref. 27, in which an array of small single superconducting annuli is cooled, and the flux passing through them measured, would provide a test of this latter prediction. Unfortunately, for single annuli we can no longer ignore flux trapping, for which the authors of Ref. 27 propose a variant, similar in spirit to that of Ref. 19, but different in detail. ${ }^{28}$ They then argue that the vortices formed by the $\mathrm{KZ}$ mechanism would, in fact, be washed out by this thermally activated vortex mechanism.

There is, however, one unexplained observation. Unlike in our earlier experiment, in this experiment we never observed the simultaneous production of two (or more) fluxons (or antifluxons), despite the fact that we have detected more than 10000 single fluxons for each sample. This requires strong correlations in the production of fluxons and antifluxons since, if defects in different ring regions are produced independently then, extrapolating from an infinite junction, the probability $f_{2}$ of trapping two homopolar fluxons is $f_{2}$ $=f_{1}^{2} / 2$. This is obviously not the case, but we do not have a model from which to make predictions.

\section{THEORY}

The value of $\sigma=0.5$ is obviously in disagreement with our earlier prediction of $\sigma=0.25$ given in Ref. 20. Two possible reasons need to be explored. The first is that the use of causality seems suspect when dealing with systems like the annuli discussed here that are smaller than the KZ causal length $\bar{\xi}$. That is, how should we treat causal horizons that are larger than the systems at the time that defects are formed, if we are using them to define defect separation? Is it the case that Eq. (3) follows from Eq. (2)? The second is that, if Eq. (3) does follow from Eq. (2), are our assumptions of idealized JTJs with weak coupling and exact critical slowing down of the
Swihart velocity at the transition used in Ref. 20 valid?

To address the first problem of seeing how Eq. (3) can arise we are helped by the fact that, since the original $\mathrm{KZ}$ papers were published, there has been considerable analytic and numerical work performed for ideal systems obeying dissipative equations, ${ }^{19,29-35}$ in particular the time-dependent Ginzburg-Landau equation. Although there has been no attempt to model Josephson tunnel junctions we can draw several conclusions for JTJs from the phase transitions in simple systems, such as superfluids and superconductors, that suggests that small system size is not a problem in principle. This requires a rather different picture from that originally proposed by Kibble and Zurek in Refs. 5-7, but with the same outcome as (2) for large systems.

As originally posed, the separation of defects is directly related to the correlation length. By definition, the correlation length is determined from the large-distance behavior of the correlation function (and thereby the position of the nearest momentum-space singularity of the power spectrum in the complex plane). On the other hand, analytic and numerical work shows that defect separation is more strongly related to the short-distance behavior of the correlation function, since it is this that controls the field zeros (in this case modulo $2 \pi$ ) with which simple defects are associated. ${ }^{36} \mathrm{In}$ the first instance after the transition this is given by the moments of the power spectrum, rather than its singularities. Of course, if there is only a single length in the model at the time the transition is implemented, the two must be essentially identical, although they play very different roles. ${ }^{35}$ Provided thermal fluctuations are controlled, this is plausible for a dissipative system. ${ }^{37}$ Field zeros, which can mature into defects, initially occur on all, particularly small, scales. Most annihilate very rapidly. What drives those that have not annihilated to become the cores of defects on large scales is the unstable growth of long-wavelength modes, which transfers power to long-distance fluctuations at the expense of small. It is a further issue as to whether the time scale for defect formation, the time it takes for long-wavelength modes to evolve fully, is related to the $\mathrm{KZ}$ causal time at which defects are formed, from which (2) follows. However, very simple arguments ${ }^{29-31}$ show that this is the case, for relatively weak couplings at least, up to logarithmic corrections. This enables us to use the KZ scenario to calculate $\sigma$ even though causality is not directly the driving mechanism.

With fluctuations starting from such small beginnings, from this viewpoint the fact that, ultimately, $\bar{\xi}>C$ does not hinder defect formation but does not, of itself, demonstrate that Eq. (3) follows from Eq. (2). However, with or without identical exponents, the undoubted scaling shown in the experiments makes the existence of a common mechanism for large and small scales compelling.

That one is a direct continuation of the other to small distances is straightforward to see. Suppose now that we increase $C$ so that $C>\bar{\xi}$ and we see fluxons every time. $f_{1}$ is then not a useful measure and, instead, we measure total flux, i.e., the variance $\Delta n$ in the net number $n$ of fluxons (i.e., the number of fluxons minus the number of antifluxons). Using the spacing of Eq. (2) a random walk in phase along the annulus suggests 


$$
(\Delta n)^{2} \approx \frac{C}{\bar{\xi}}=\frac{C}{\xi_{0}}\left(\frac{\tau_{Q}}{\tau_{0}}\right)^{-\sigma} .
$$

Since $\langle n\rangle=0,(\Delta n)^{2}=\left\langle n^{2}\right\rangle=f_{1}$ when $f_{1}<1$ is sufficiently small that we can neglect the possibility of seeing more than one fluxon. As a result Eq. (3) is the continuation of Eq. (2), without having to use causality directly.

More work is being done, particularly on simple numerical simulations with periodic boundary conditions, ${ }^{38}$ but to date we see no mechanism for a change in exponent $\sigma$ because of small size. In fact, there is no need for a change in $\sigma$ between large and small annuli since there are other reasons, concerning the specific properties of the JTJs, that can provide an explanation for $\sigma=1 / 2$. To reiterate, the theory in Ref. 20 was developed for JTJs whose electrodes are weakcoupling superconductors for which the temperature dependence of the critical current density $J_{c}(T)$ is given by the Ambegaokar-Baratoff equation ${ }^{39}$

$$
J_{c}(T)=\frac{\pi}{2} \frac{\Delta(T)}{e \rho_{N}} \tanh \frac{\Delta(T)}{2 k_{B} T},
$$

where $\Delta(T)$ is the superconducting gap energy and $\rho_{N}$ is the JTJ normal resistance per unit area. Equation (7) provides a linear decrease of $J_{c}$ near $T_{c}$ :

$$
J_{c}(T)=\alpha J_{c}(0)\left(1-\frac{T}{T_{c}}\right),
$$

in which the dimensionless quantity $\alpha$ is approximately equal to $2 \Delta(0) / k_{B} T_{C}=3.5$. However, our JTJs are based on $\mathrm{Nb}$, a strong-coupling superconductor, for which Eq. (7) is not necessarily valid. In practice, high-quality and reproducible barriers are achieved by depositing a thin Al overlay onto the $\mathrm{Nb}$ base electrode which will be only partially oxidized, leaving a $\mathrm{Nb}$-Al bilayer underneath having a non-BCS temperature dependence of the energy gap and of the density of states. The proximity effect in $\mathrm{Nb}-\mathrm{Al} / \mathrm{AlO}_{x} / \mathrm{Nb}$ JTJs has been extensively studied and it is known to influence the electrical properties of the junctions, such as the currentvoltage characteristic and the temperature dependence of the critical current density. Specifically, the proximity effect in $\mathrm{S}-\mathrm{N}-\mathrm{I}-\mathrm{S}$ junctions is responsible for an otherwise subdominant temperature dependence of the critical current density ${ }^{40}$ dominating in the vicinity of $T_{c}$ as

$$
J_{c}(T) \simeq \alpha^{\prime} J_{c}(0)\left(1-\frac{T}{T_{c}}\right)^{2},
$$

where $\alpha^{\prime}$ is a constant depending on the degree of proximity. The last equation models the tail-shaped dependence of $J_{c}$ vs $T$ near $T_{c}$; it has been theoretically derived and experimentally confirmed by Golubov et al. ${ }^{41}$ in 1995.

Assuming that the proximity effect is important for our samples here then, on rephrasing the arguments of Ref. 20 with Eq. (9) replacing Eq. (8), the Josephson penetration depth $\lambda_{J}(T)$, which plays the role of the system equilibrium coherence length $\xi(T)$, now diverges linearly near $T_{c}$ as

$$
\xi(T)=\lambda_{J}(T) \propto J_{c}(T)^{-1 / 2}=\xi_{0}\left(1-\frac{T}{T_{c}}\right)^{-1},
$$

where

$$
\xi_{0}=\sqrt{\frac{\hbar}{2 e \mu_{0} d_{s} \alpha^{\prime} J_{c}(0)}},
$$

$d_{s}$ being the electrode thickness.

At time $t$ close to the transition, the temperature $T(t)$ satisfies

$$
\left(1-\frac{T(t)}{T_{c}}\right) \simeq \frac{t}{\tau_{Q}}
$$

where $T(0)=T_{c}$. The first assumption in the KZ scenario is that causality establishes a time $\bar{t}$ at which domains and defects form, at which time defect separation is $\xi(T(\vec{t}))$. For JTJs fluxon separation is, from (10),

$$
\bar{\xi}=\xi(T(\bar{t}))=\lambda_{J}(T(\bar{t}))=\xi_{0} \frac{\tau_{Q}}{\bar{t}},
$$

instead of the $\xi_{0} \sqrt{\tau_{Q} / \bar{t}}$ behavior of Ref. 20 that we used in Refs. 1 and 2 .

We have argued that, although it is instability, rather than direct causality, which drives scaling behavior, it arises in a way that is quantitatively indistinguishable from the KZ scenario.

Thus, even where causality is an inappropriate mechanism we can still adopt its results, that the earliest possible time $t$ at which defects could possibly appear satisfies

$$
\dot{\xi}(\bar{t})=-\bar{c}(\bar{t}),
$$

where $\bar{t}$ is the causal time and $\bar{c}$ is the Swihart velocity.

As we said, in Ref. 20 we had assumed that the Swihart velocity vanished at $T_{c}$ whereas, for realistic JTJs, it just becomes very small. Swihart ${ }^{21}$ has demonstrated that for a thin-film superconducting strip transmission line the solution for the velocity varies continuously as one passes through the critical temperature into the normal state. As a result, we assume $\bar{c}(t)=\bar{c}_{n n}$ near the transition temperature where $\bar{c}_{n n}$ is the speed of light in a microstrip line made of normal metals. In the case of a microstrip line made by two electrodes having the same thickness $d_{s}$ and the same skin depth $\delta$, with $d_{s} \ll \delta$, separated by a dielectric layer of thickness $d_{o x}$ and dielectric constant $\epsilon$, its value,

$$
\bar{c}_{n n}=\frac{2}{\delta} \sqrt{\frac{d_{o x} d_{s}}{\epsilon \mu}},
$$

depends on the temperature very weakly, but depends on the frequency $f$ through $\delta=\sqrt{\rho / \pi \mu f}, \rho$ being the normal metal residual resistivity.

The match $\bar{c}\left(T_{c}\right)=\bar{c}_{n n}$ is certainly realistic and we still have approximate critical slowing down insofar as $\bar{c}_{n n}$ is much smaller than the zero-temperature Swihart velocity $\bar{c}_{0}$ $=\sqrt{d_{o x} / 2 \lambda_{L 0} \epsilon \mu}$, i.e., when the zero-temperature London penetration depth $\lambda_{L 0} \ll \delta^{2} / d_{s}$. For 300-nm-thick $\mathrm{Nb}$ electrodes $\left(\rho=3.8 \mu \Omega \mathrm{cm}\right.$ and $\left.\lambda_{L 0}=90 \mathrm{~nm}\right), \quad \delta \simeq 1 \mathrm{~mm}$ at, say, 
$f=10 \mathrm{kHz}$, so the last inequality is fully satisfied. At the same frequency, for a value of the specific barrier capacitance $c_{s}=\epsilon / d_{o x}=0.02 \mathrm{~F} / \mathrm{m}^{2}$ typical of low-current-density $\mathrm{Nb}-\mathrm{Al} / \mathrm{AlO}_{x} / \mathrm{Nb}$ JTJs, we get $\bar{c}_{n n}=7 \times 10^{3} \mathrm{~m} / \mathrm{s}$ and $\bar{c}_{0}=1.4$ $\times 10^{7} \mathrm{~m} / \mathrm{s}$. We stress that $\bar{c}_{n n}$ is finite and orders of magnitude smaller than the zero-temperature Swihart velocity $\bar{c}_{0}$.

The solution of the causality equation (14) with a nonvanishing Swihart velocity yields a new expression for the socalled Zurek or freeze-out time $\bar{t}=\sqrt{\xi_{0} \tau_{Q} / \bar{c}_{n n}}=\sqrt{\tau_{0} \tau_{Q}}$, where $\tau_{0}=\xi_{0} / \bar{c}_{n n}\left[\tau_{0}=O(1 \mathrm{~ns})\right]$.

Inserting the value of $\bar{t}$ into Eq. (10) we obtain the new Zurek length $\bar{\xi}$ :

$$
\bar{\xi}=\xi(\bar{t})=\sqrt{\xi_{0} \tau_{Q} \bar{c}_{n n}}=\xi_{0}\left(\frac{\tau_{Q}}{\tau_{0}}\right)^{1 / 2} .
$$

We reach the important conclusion for realistic JTJs that the probability $f_{1}$ for spontaneously producing one fluxon in the quench is still predicted to scale with the quench time $\tau_{Q}$ according to Eq. (3), but the critical exponent is now $\sigma$ $=0.5$, rather than $\sigma=0.25$.

By varying $\tau_{Q}$ in the experimentally achieved four-decade range $1 \mathrm{~ms}<\tau_{Q}<10 \mathrm{~s}$, we get $10 \mu \mathrm{s}<\bar{t}<1 \mathrm{~ms}$, which is always much larger than $\tau_{0}$; it means that by the time the Josephson phase freezes the Josephson effect is well established.

The freezing temperature $\bar{T}=T(\bar{t})=T_{c}\left(1-\bar{t} / \tau_{Q}\right)$ comes out to differ from the critical temperature itself by an amount $T_{c}-\bar{T}=T_{c} \sqrt{\tau_{0} / \tau_{Q}}$. Further, in the same $\tau_{Q}$ range the normalized freezing temperature $\bar{T} / T_{C}$ at which the defect is formed is $0.99<\bar{T} / T_{C}<0.9999$. It would be really hard, if not impossible, to measure the temperature dependence of $J_{c}$ and $\bar{c}$ so close to $T_{C}$. We have then to resort to theoretical predictions, that is, to Eq. (9), for the temperature dependance of the Josephson current density $J_{c}(T)$. Further, according to Swihart's calculations and figures, ${ }^{21}$ we are allowed to assume $\bar{c}(t)=c_{n n}$ near the transition temperature.

\section{CONCLUSIONS}

Equation (3) is amenable to further experimental tests with AJTJs having different critical current densities $J_{c}(0)$ and/or circumferences $C$. Such experiments should still show the critical exponent $\sigma=1 / 2$; however, the prefactor should change accordingly. According to the theory presented in this paper, the expected dependence of the single-fluxon trapping frequency on the critical current is weak, being proportional to its fourth root, but critical current densities 20-30 times larger should produce detectable effects. The change in trapping frequency will be more easily to observe with larger diameter annuli due to the linear dependence on $C$ in Eq. (3). Another test of importance is provided by working with asymmetric Nb-AlN-NbN junctions for which we would expect a smaller value for $\sigma$. Such experiments are in process of being performed.
It is worth commenting here on the effect of the unavoidable thermal gradients in physical system undergoing a thermal quench; the Zurek-Kibble scaling law Eq. (3) was derived assuming that thermal gradients are not a problem. More precisely, according to the general theory of Ref. 42, the maximum thermal gradient $\nabla T^{*}$ allowed across a given system undergoing a thermal quench at the time of the transition amounts to $\left(\bar{T}-T_{C}\right) / \bar{\xi}$. Below this threshold a saturation is expected in the spontaneous production of defects. In particular, in our case, where $\tau_{Q}$ ranges in the interval $10 \mathrm{~s}-1 \mathrm{~ms}$, we have $\nabla T^{*}=T_{C} /\left(\bar{c}_{n n} \tau_{Q}\right)$ ranges from $6.5 \mu \mathrm{K} / \mathrm{mm}$ to $65 \mathrm{mK} / \mathrm{mm}$ across our $160 \mu \mathrm{m}$-diameter ring corresponding, to a critical value for the maximum temperature difference $\Delta T_{\max }=\nabla T^{*} \times C / \pi \approx 1 \mu \mathrm{K}-10 \mathrm{mK}$. As stated before, our voltage accuracy in the measurements of the gap voltage in contiguous JTJs on the same chip allows us to resolve temperature differences as small as a few millikelvin; the simultaneous measure of the time dependence of the gap voltages of contiguous junctions in response to the shortest heat pulse (i.e., corresponding to $\tau_{Q}=1 \mathrm{~ms}$ ) revealed that the temperature difference, if any, is less than $10 \mathrm{mK}$. Further, the fact that we have not observed any saturation of the defect production in a wide $\tau_{Q}$ range should indicate that thermal gradient still do not affect the defect production in our experiments. Again, in future experiments devised to understand the role of the thermal gradients, they can be enhanced by progressively reducing the thermal coupling between the chip and its surroundings and/or by supplying the voltages pulses only to one of the two resistive stripes integrated at the chip extremities. On the opposite, if needed, it is also possible to further reduce the thermal gradients by using an annular integrated heater superimposed on and concentric to the annular JTJ.

In summary, we see this experiment as demonstrating unambiguous scaling over a wide range of quenching time $\tau_{Q}$. Moreover, we can understand the scaling exponent for $\mathrm{Nb}-\mathrm{Al} / \mathrm{AlO}_{x} / \mathrm{Nb}$ JTJs in terms of their properties within the framework of the KZ scenario. As such, it replaces the experiment reported in Refs. 1 and 2 by being more realistic theoretically and more sophisticated experimentally. To our knowledge, we are the only group to have reliably confirmed scaling for a condensed matter system. We note, however, that scaling has been observed ${ }^{43}$ in nonlinear optical systems, which satisfy equations of the time-dependent LandauGinzburg type.

\section{ACKNOWLEDGMENTS}

The authors thank P. Dmitriev, A. Sobolev, and M. Torgashin for the sample fabrication and testing and U. L. Olsen for the help at the initial stage of the experiment. This work is, in part, supported by the COSLAB program of the European Science Foundation, the Danish Research Council, the Hartmann Foundation, the RFBR Project No. 06-02-17206, and the Grant for Leading Scientific School No. 7812.2006.2. 
*Electronic address: roberto@sa.infn.it

Electronic address: aaroe@fysik.dtu.dk

Electronic address: r.rivers@imperial.ac.uk

§Electronic address: valery@ hitech.cplire.ru

${ }^{1}$ R. Monaco, J. Mygind, and R. J. Rivers, Phys. Rev. Lett. 89, 080603 (2002)

${ }^{2}$ R. Monaco, J. Mygind, and R. J. Rivers, Phys. Rev. B 67, 104506 (2003).

${ }^{3}$ R. Monaco, J. Mygind, M. Aaroe, R. J. Rivers, and V. P. Koshelets, Phys. Rev. Lett. 96, 180604 (2006).

${ }^{4}$ T. W. B. Kibble, J. Phys. A 9, 1387 (1976).

${ }^{5}$ T. W. B. Kibble, Phys. Rep. 67, 183 (1980).

${ }^{6}$ W. H. Zurek, Nature (London) 317, 505 (1985); Acta Phys. Pol. B 24, 1301 (1993).

${ }^{7}$ W. H. Zurek, Phys. Rep. 276, 177 (1996).

${ }^{8}$ R. Jeannerot, J. Rocher, and M. Sakellariadou, Phys. Rev. D 68, 103514 (2003).

${ }^{9}$ I. Chuang, R. Durrer, N. Turok, and B. Yurke, Science 251, 1336 (1991).

${ }^{10}$ S. Digal, R. Ray, and A. M. Srivastava, Phys. Rev. Lett. 83, 5030 (1999).

${ }^{11}$ P. C. Hendry, N. S. Lawson, R. A. M. Lee, P. V. E. McClintock, and C. D. H. Williams, Nature (London) 368, 315 (1994).

${ }^{12}$ M. E. Dodd, P. C. Hendry, N. S. Lawson, P. V. E. McClintock, and C. D. H. Williams, Phys. Rev. Lett. 81, 3703 (1998); J. Low Temp. Phys. 15, 89 (1999).

${ }^{13}$ C. Bauerle, Y. M. Bunkov, S. N. Fisher, H. Godfrin, and G. R. Pickett, Nature (London) 382, 332 (1996).

${ }^{14}$ V. M. H. Ruutu, V. B. Eltsov, A. J. Gill, T. W. B. Kibble, M. Krusius, Y. G. Makhlin, B. Placais, and G. E. Volovik, Nature (London) 382, 334 (1996).

${ }^{15}$ R. Carmi and E. Polturak, Phys. Rev. B 60, 7595 (1999).

${ }^{16}$ A. Maniv, E. Polturak, and G. Koren, Phys. Rev. Lett. 91, 197001 (2003).

${ }^{17}$ R. Carmi, E. Polturak, and G. Koren, Phys. Rev. Lett. 84, 4966 (2000).

${ }^{18}$ S. Casado, W. González-Viñas, H. Mancini, and S. Boccaletti, Phys. Rev. E 63, 057301 (2001).

${ }^{19}$ M. Hindmarsh and A. Rajantie, Phys. Rev. Lett. 85, 4660 (2000); A. Rajantie, J. Low Temp. Phys. 124, 5 (2001).

${ }^{20}$ E. Kavoussanaki, R. Monaco, and R. J. Rivers, Phys. Rev. Lett. 85, 3452 (2000); R. Monaco, R. J. Rivers, and E. Kavoussanaki,
J. Low Temp. Phys. 124, 85 (2001).

${ }^{21}$ J. C. Swihart, J. Appl. Phys. 32, 461 (1961).

${ }^{22}$ A. Barone and G. Paterno, Physics and Applications of the Josephson Effect (John Wiley \& Sons, New York, 1982).

${ }^{23}$ P. N. Dmitriev, I. L. Lapitskaya, L. V. Filippenko, A. B. Ermakov, S. V. Shitov, G. V. Prokopenko, S. A. Kovtonyuk, and V. P. Koshelets, IEEE Trans. Appl. Supercond. 13, 107 (2003).

${ }^{24}$ D. J. Thouless, Phys. Rev. 117, 1256 (1960).

${ }^{25}$ R. F. Broom, J. Appl. Phys. 47, 5432 (1976).

${ }^{26}$ R. Monaco, J. Mygind, M. Aaroe, V. P. Koshelets, and R. J. Rivers (unpublished).

${ }^{27}$ J. R. Kirtley, C. C. Tsuei, and F. Tafuri, Phys. Rev. Lett. 90, 257001 (2003).

${ }^{28}$ T. W. B. Kibble and A. Rajantie, Phys. Rev. B 68, 174512 (2003).

${ }^{29}$ G. Karra and R. J. Rivers, Phys. Lett. B 414, 28 (1997).

${ }^{30}$ E. Moro and G. Lythe, Phys. Rev. E 59, R1303 (1999).

${ }^{31}$ R. J. Rivers, J. Low Temp. Phys. 124, 41 (2001).

${ }^{32}$ P. Laguna and W. H. Zurek, Phys. Rev. D 58, 085021 (1998); Phys. Rev. Lett. 78, 2519 (1997).

${ }^{33}$ N. D. Antunes, L. M. A. Bettencourt, and W. H. Zurek, Phys. Rev. Lett. 82, 2824 (1999).

${ }^{34}$ D. Ibaceta and E. Calzetta, Phys. Rev. E 60, 2999 (1999).

${ }^{35}$ N. D. Antunes, P. Gandra, and R. J. Rivers, Phys. Rev. D 73, 125003 (2006)

${ }^{36}$ B. I. Halperin, in Physics of Defects, edited by R. Balian, L. Kléman, and R. Poirier, Proceedings of the Les Houches Summer School for Theoretical Physics, XXXV (North-Holland, Amsterdam, 1981), p. 816; F. Liu and G. F. Mazenko, Phys. Rev. B 46, 5963 (1992); A. J. Gill and R. J. Rivers, Phys. Rev. D 51, 6949 (1995).

${ }^{37}$ G. Karra and R. J. Rivers, Phys. Rev. Lett. 81, 3707 (1998).

${ }^{38}$ P. Gandra, R. J. Rivers, and A. Swarup (unpublished).

${ }^{39}$ V. Ambegaokar and A. Baratoff, Phys. Rev. Lett. 10, 486 (1963); 11, 104(E) (1963).

${ }^{40}$ N. L. Rowell and H. J. T. Smith, Can. J. Phys. 54, 223 (1976).

${ }^{41}$ A. A. Golubov, E. P. Houwman, J. G. Gijsbertsen, V. M. Krasnov, J. Flokstra, H. Rogalla, and M. Yu. Kupriyanov, Phys. Rev. B 51, 1073 (1995).

${ }^{42}$ T. W. B. Kibble and G. E. Volovik, Pis'ma Zh. Eksp. Teor. Fiz. 65, 96 (1997) [JETP Lett. 65, 102 (1997)].

${ }^{43}$ S. Ducci, P. L. Ramazza, W. González-Viñas, and F. T. Arecchi, Phys. Rev. Lett. 83, 5210 (1999). 